\title{
Educación inclusiva
}

con enfoque comunitario: fórmula para afianzar la convivencia en contextos de significativa diversidad cultural

Dr. Vicente Manuel Zapata Hernández Departamento de Geografia e Historia

Universidad de La Laguna vzapata@ull.es 


\section{Edita: Observatorio de la Inmigración de Tenerife (OBITen)}

Título: Educación inclusiva con enfoque comunitario: fórmula para afianzar la convivencia en contextos de significativa diversidad cultural

Autor: Vicente Manual Zapata Hernández

\section{Edición: mayo de 2019}

Diseño y maquetación: Javier Cabrera S.L

DOI: https://doi.org/10.25145/r.obitfact.2019.06 
El sistema educativo y el ecosistema que conforma con sus múltiples actores y ramificaciones, constituye uno de los recursos fundamentales para afianzar los procesos de convivencia social en contextos de significativa diversidad cultural, resultado de la amplificación de las migraciones internacionales. Esto implica mayor apertura y permeabilidad hacia su marco socioterritorial de referencia, donde la comunidad debe saber identificar y aprovechar las oportunidades que se derivan de planteamientos más integradores e inclusivos de la Educación en sus distintos niveles. El enfoque intercultural propicia ahondar en el valor de las relaciones y de la reformulación permanente de una identidad colectiva, construida a partir de las múltiples aportaciones, siendo la propia diversidad uno de sus pilares fundamentales. Confluencia beneficiosa y con efectos ciertos en los centros y sus respectivos contextos, sobre todo si las iniciativas se plantean con objetivos de permanencia y adquieren carácter estructural, además de ser construidas de manera participada y con perspectiva de alentar procesos transformadores. 

T as migraciones internacionales suponen un fenómeno global cuya intensiUficación y diversificación recientes generan renovados y complejos retos a las sociedades, tanto en el origen como en el destino y en las áreas de tránsito de los desplazamientos. Retos particularmente centrados en contextos locales, a veces poco relacionados con las principales causas que provocan dicha movilidad, pero afectados por sus múltiples consecuencias e implicaciones, siendo la esfera de la cohesión y convivencia sociales ámbitos de significativo impacto, sobre todo allí hacia donde se concentra el asentamiento de las personas recién llegadas.

Y es que, el creciente carácter multicultural de muchos territorios y una mayor complejidad social, implican afrontar lo que se conoce por gestión de la diversidad cultural, con el horizonte de afianzar procesos de integración y el objetivo de consolidar contextos generales de convivencia en los que las personas puedan encontrar respuestas a sus múltiples necesidades y aspiraciones. Algunos sistemas de amplia cobertura socioterritorial pueden contribuir efectivamente al desarrollo y consolidación de dichos procesos de una manera más que relevante, como es el caso del sistema educativo.

De hecho, una de las claves para asumir con garantías esa nueva realidad socioterritorial, que implica el ascenso de la diversidad en el contexto local, aunque con proyección a escalas superiores o territorios más amplios, es la apertura y la competencia de los sistemas educativos, sobre todo por sus conexiones y ramificaciones hacia múltiples instituciones y actores, tanto públicos como privados. Desde la Administración hasta el ámbito familiar, la ciudadanía y sus organizaciones representativas, las entidades sociales y otros sistemas como el sanitario (salud pública) o el asistencial, el que atiende los requerimientos de empleabilidad y empleo, etc.

La comunidad educativa constituye un organismo complejo y dinámico, integrado por sus protagonistas directos, alumnado y profesorado, más otro personal de apoyo y las personas responsables de su gestión a distintos niveles institucionales, así como las familias con mayor o menor grado de organización e implicación (Asociaciones de Madres y Padres o AMPAS, por ejemplo). Organismo que interactúa de manera permanente con múltiples actores clave, o podría hacerlo, como ya se ha señalado, pudiendo tener una influencia capital en el entorno social en el que se proyecta, asumiendo un importante papel en su proceso de transformación y mejora. 
Esa capacidad de influencia se adquiere con el tiempo, si se consigue abrir el sistema y cada uno de sus nodos a la realidad del entorno que se atiende - tanto el sistema público de manera directa, como atrayendo de forma progresiva al concertado y al privado-, particularmente con enfoque comunitario y en clave de tejer estrategias inclusivas que atiendan la diversidad con perspectiva intercultural. Enfoque comunitario que facilite la necesaria permeabilidad para construir proyectos sociales integradores con la participación de todos sus protagonistas, a partir del basamento que proporcionan los centros educativos en sus distintos niveles, desde la escuela hasta el grado superior.

Y perspectiva intercultural para avanzar hacia el establecimiento de relaciones multidireccionales que conformen escenarios de convivencia, superando así ambientes de hostilidad o contextos de insuficiente coexistencia, como señalaría Carlos Giménez. Interculturalidad como paradigma para la construcción de nuevas realidades colectivas y renovadas identidades, desde la conjugación de todas las aportaciones originales que van llegando, sin renunciar a la permanencia de sus identificadores, haciendo visible el valor de la diversidad como palanca de progreso y cambio desde el aprovechamiento de las experiencias múltiples: la diversidad hace la fuerza.

¿En qué barrio o pueblo o en sus proximidades no existe un centro educativo? La Educación constituye un sistema con plena o significativa cobertura socioterritorial en muchos lugares, organizado a partir de centros o nodos que se transforman en fuente de potencial irradiación hacia su entorno, llegando a buena parte del conjunto poblacional a través de las familias, y también a la comunidad - concepto más amplio y complejo que el primero-, si logra convertirse en referencia fundamental para avanzar en temas del máximo interés social como es la convivencia. Sistema que tiene además la capacidad de incorporar casi de manera inmediata a lo nuevo, por su relación con ello a través de la escolarización o de las necesidades formativas permanentes de las personas.

Lo nuevo representado por las personas y familias recién llegadas, las nuevas manifestaciones de la diversidad en lo concerniente a modos de vida y costumbres, culturas y formas de relación, ideas y aprendizajes, maneras de entender la realidad y el desarrollo futuro, encajándolo en un contexto de comprensión, respeto y construcción compartida necesario para que fructifique la fusión. Regresamos entonces a la premisa de la permeabilidad, al fundamento de la conformación de estructuras inclusivas y perdurables, particularmente construidas desde la esfera educativa aprovechado su centralidad en la vida de las personas, a veces durante prácticamente toda nuestra existencia.

Y todo ello implica reforzar la apertura y conexión del sistema en relación con entornos dinámicos, que evolucionan en muchos casos a un ritmo que no es sencillo seguir, pero para lo que hay que prepararse y preparar al resto, aprovechando el ir y venir de saberes, ideas, recursos, propuestas e intenciones; aportaciones que se convierten en indispensables ingredientes para armar estrategias transformadoras, desde lo personal a lo colectivo, avanzando siempre 
hacia lo comunitario. Estrategias que inspiren proyectos educativos que vayan más allá del aula y del centro, siendo su marco de referencia el contexto socioterritorial en el que se proyecta la comunidad educativa; y por qué no, los territorios de origen y tránsito de la diversidad existente. La compleja realidad compartida.

Porque parece que se ha avanzado bastante en lo concerniente a la generación de contextos educativos inclusivos, integradores, promoviendo estrategias y proyectos que abonan la semilla de la convivencia y cuidan de su florecimiento, pero que, no siempre, conforman procesos que transcienden de los recintos escolares. Siendo importante ese avance, no es suficiente y se desaprovecha en buena medida el potencial ya descrito, y que, además, requiere para su plena fructificación de la adecuación del contexto social. Ahí se identifica el reto fundamental: trascender de lo personal a lo colectivo, de lo particular a lo general, de lo sectorial a lo global, como diría Marco Marchioni. Del aula a la calle.

¿Cómo? Sin desviar la atención hacia el interior, debemos asimismo mirar hacia afuera, considerando el contexto socioterritorial de referencia. Para comenzar, identificando otras dinámicas, procesos o iniciativas específicas que se estén desarrollando, para conocerlas y aprovechar su existencia si se alinean con nuestro objetivo integrador. Eso implica la generación de nuevas competencias en el seno de la comunidad educativa, que permitan realizar esa labor de reconocimiento, relación y concertación, con la intención de definir nuevas estrategias compartidas por un mayor número de actores. Estrategias con aspiración transformadora, por lo tanto, perdurables en el tiempo y sostenibles.

Sostenibilidad que habitualmente le confieren un planteamiento coral y una expresión en red. Estrategias formuladas de manera participativa e inspiradas en principios generales que puedan ser asumidos por sus protagonistas en clave de proceso, por ejemplo, basados en los objetivos de desarrollo sostenible (ODS), en cuya pizarra podemos encontrar argumentos sólidos para afianzar esta propuesta, que asimismo persiguen provocar un cambio positivo en las personas y en el planeta: educación de calidad (4), ciudades y asentamientos humanos inclusivos, seguros, resilientes y sostenibles (11), sociedades justas, pacíficas e inclusivas (16), alianzas para lograr objetivos (17). Magnífico baúl para encontrar claves reveladoras.

Hablamos de nuevas competencias para identificar necesidades y oportunidades en el contexto socioterritorial desde la esfera educativa, relacionadas con la convivencia y sus derivadas, a partir de una labor que pueda ser asumida por un número mayor de actores de la propia comunidad educativa, que permita escrutar la realidad con una mirada generosa e incluyente. Lente que permita indagar tanto a profesorado y equipos directivos - deben ser cada vez mejor reflejo de la propia diversidad existente-, como a alumnado y familias, junto a las instituciones con presencia en el devenir educativo. Mejor, conjuntamente. Se reconocen así potenciales ámbitos de intervención y se generan estrategias para concurrir al lado de sus protagonistas. 
Conectado con la idea de permeabilidad antes mencionada, la propuesta que se formula requiere el surgimiento de más espacios de encuentro y relación entre la comunidad educativa y el contexto general; espacios y estructuras facilitadoras, motivadoras y hasta mediadoras. Por una parte, esto implica más apertura de los centros a todos los niveles, incluyendo la posibilidad de convertirse en una dotación de referencia para la comunidad de manera compatible con las funciones educativas; y por otra parte, la proyección de la dinámica educativa en el entorno, no como hechos puntuales, sino como una práctica habitual ampliando su marco de desarrollo hacia el espacio público, centros ciudadanos, recintos culturales, instalaciones vecinales, etc.

Y ese continuo viaje de ida y de vuelta debe producir impactos múltiples, hacia el interior del sistema educativo y hacia su entorno, hacia las personas directamente implicadas y hacia el conjunto social, hacia los particulares y hacia las instituciones. Es el objetivo, y al mismo tiempo, la referencia para otros sistemas que también deberían realizar similar recorrido, puesto que lo sectorial debe aspirar a confluir y propiciar una visión más integral e integrada de la realidad; integradora además de la diversidad existente como estrategia para afianzar la convivencia en contextos de diversidad.

Entre los impactos o efectos que se producen, son evidentes los que atañen a la institución y al proceso educativo en sus distintos niveles y fases. Ricos aprendizajes entroncados en los principales retos sociales; institución educativa más comprometida con sus circunstancias socioterritoriales, lo que supone una mejora apreciable de su consideración y hasta de su reputación; más oportunidades de intervención por parte de los componentes de la comunidad educativa, que pueden participar activamente de las estrategias definidas; incorporación de experiencias gratificantes y motivadoras por conectar con necesidades y aspiraciones reales de las personas y las organizaciones, etc.

En síntesis, no se puede concebir la integración y la atención a la diversidad desde el exclusivo cuidado de la convivencia en los centros educativos, desconectados del contexto socioterritorial en que se proyectan. Para que estos procesos fructifiquen se debe contemplar en su integridad la dinámica vital de las personas, su día a día, que va más allá del tiempo de escolarización o formación; cabe soñar entonces con incorporar su completo ciclo y experiencia personal, lo que entroncaría con contextos geográficos y marcos temporales más amplios. Y en esa necesidad se produce el encuentro de la esfera educativa con la comunidad en la que se inserta, que requiere asimismo modelos de referencia y firmes anclajes para orientar el proceso de convivencia general, conjugando los esfuerzos sectoriales. El sistema educativo los puede propiciar, aunque debe prepararse para ello. 


\section{DR. VICENTE MANUEL ZAPATA HERNÁNDEZ}

Geógrafo. Profesor titular de Geografia Humana de la Universidad de La Laguna. Promotor y director del Observatorio de la Inmigración de Tenerife (OBITen). Responsable de los proyectos Juntos En la misma dirección, Intervención Comunitaria Intercultural ICI Taco y Barrios por el Empleo: Juntos más Fuertes.

Proyectos locales de referencia en los que ha participado el autor del documento en los últimos años, relacionados con la temática que se aborda:

'Intervención experimental en materia de empleabilidad en Comunidades Educativas' y 'Fomento de la empleabilidad de personas desempleadas en el entorno de las Comunidades Educativas' (ICE) Proyecto Barrios por el Empleo: Juntos más Fuertes

\section{http://bxe.es}

'Escuela Abierta de Verano Yo soy Taco'

'Programa social de apoyo familiar al éxito educativo'

Proyecto Intervención Comunitaria Intercultural (ICI Taco)

\section{http://www.yosoytaco.org/icitaco}

'Grupo de Trabajo de Educación para el Desarrollo'

'Grupo de Trabajo de Participación Infantil Amiguitos y Amiguitas' Proyecto Juntos En la misma dirección

\section{http://www.juntosenlamismadireccion.com}

'Análisis de la realidad del CEIP Guayonge y propuestas de actuación a partir de las aportaciones de la comunidad educativa (2016-2017)' AMPA Guayonsete y CEIP Guayonge, Tacoronte, Tenerife, Islas Canarias. 


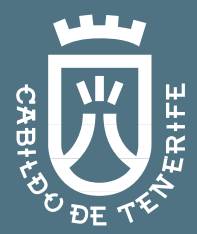

$\checkmark$ Universidad

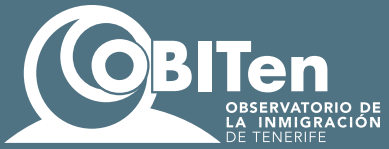

